\title{
MODELAGEM MOLECULAR DA DESCARBOXILAÇÃO ASSISTIDA POR ÁGUA E POR MICELAS
}

\author{
Stephanie Borges Vieira ${ }^{1 *}$, Diego de Paula Santos², Ricardo Luiz Longo ${ }^{3}$, \\ ${ }^{1}$ Laboratório de Química Teórica e Computacional, Departamento de Química Fundamental, UFPE \\ *stephanieborgesv@gmail.com
}

\section{INTRODUÇÃO}

Neste trabalho foi realizado um estudo sobre as reações de descarboxilação de aminoácidos no interior da micela SB3-14, que consiste num monômero de sulfobetaína com 14 carbonos em sua cadeia alifática e 3 carbonos na cadeia entre os grupos amônio e sulfonato. Este surfactante possui carga positiva interna (amônio) e carga negativa mais externa (sulfonato) conforme a Figura 1. Essas reações ocorrem através da perda de $\mathrm{CO}_{2}$ de um grupo carboxila (ou carboxilato) pelo rompimento da ligação $\mathrm{C}^{-} \mathrm{COO}^{-}$para gerar a amina correspondente.

As reações de descarboxilação possuem bastante relevâ ncia acadêmica, biológica e industrial devido ao fato de estarem presentes no metabolismo dos seres vivos, na preparação de diversos fármacos, em produtos químicos industriais, entre outros. 0 fato da descarboxilação espontânea de aminoácidos ser uma das reações mais lentas conhecidas é uma vantagem evolutiva, pois tornam os polipeptídios muito estáveis. As micelas aumentam a velocidade da reação de descarboxilação e, aquelas formadas pelos surfactantes SB3-14 ou SBPr3-14, por exemplo, aumentam em 220 e 1100 vezes, respectivamente, a velocidade da descarboxilação do ânion 6-NBIC (6,7-dimetilbenzo[d]isoxazol-3-carboxilato) em água quando comparada com a reação na ausência das micelas.

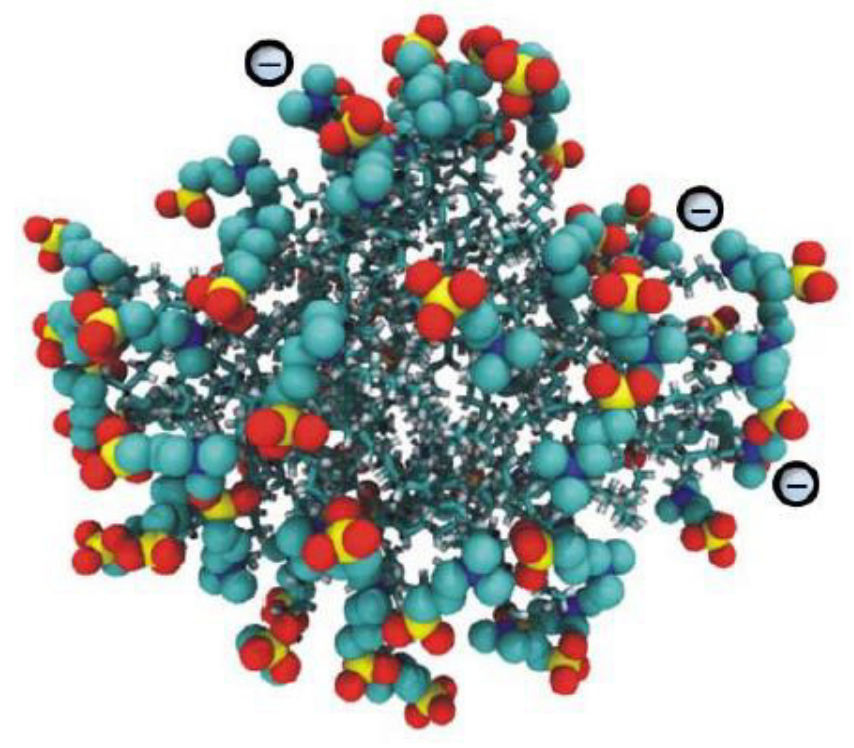

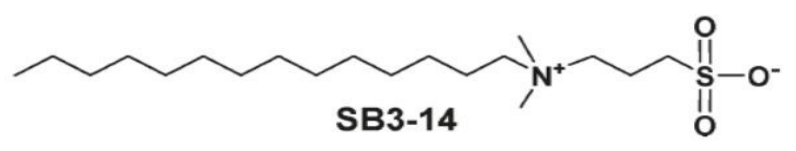

Figura 1. Estrutura da micela SB3-14 em água (painel superior). Para facilitar a visualizac ão as moléculas de água estão ocultas. Estrutura do surfactante $N$-tetradecil- $N, N$-dimetil3-amonio-1-propanosulfonato (SB3-14).

Os sistemas biológicos contêm membranas e vesículas formadas por surfactantes zwitteriônicos que, de maneira similar à s micelas zwitteriônicas (SB3-14 e SBPr3-14), podem causar uma reação de descarboxilação espontânea de aminoácidos em água. Por isso, neste trabalho, será realizado um estudo da reação de descarboxilação da glicina em água com diferentes métodos (semiempíricos PDDG/PM3, DFT com funcional B3LYP e métodos perturbativos MP2). Para quantificar o efeito da micela zwitteriô nica foi realizada a adsorção da glicina $\left(\mathrm{H}_{3} \mathrm{~N}^{+}-\mathrm{CH}_{2}-\mathrm{COO}^{-}\right)$na micela SB3-14 em água com o método de dinâmica molecular. Estamos realizando testes com íons na micela para aumentar o tempo de permanência da glicina na micela.

\section{MATERIAIS E MÉTODOS}

Para modelar a descarboxilação da forma zwitteriônica da glicina em água foram utilizados métodos de química quântica semiempíricos (PDDG/PM3), funcional da densidade (DFT), ab initio (MP2) e por QM/MM. 0 modelo inclui moléculas de água para assistir a descarboxilação e subsequente migração do próton para a formação da metilamina. Nesse caso, para os métodos B3LYP e MP2, o perfil de reação apresentou estruturas para os reagentes, produtos e prováveis estados de transição. Na etapa de dinâmica molecular foram utilizados os parâmetros da glicina na forma zwitteriônica e construiu-se a caixa de simulação com moléculas de água e a molécula de glicina no centro. 0 sistema foi minimizado em 10000 passos e posteriormente equilibrado no ensemble NPT a fim de obter a densidade correta para então calcular as médias das propriedades num ensemble NVT. Com os resultados obtidos nesta etapa observou-se que os valores de temperatura e pressão estão na média dos valores inicialmente estabelecidos ( $1 \mathrm{~atm}$ e $300 \mathrm{~K}$ ). 0 número de águas de solvatação é aproximadamente igual a três e está em concordância com os valores experimentais.

A estrutura equilibrada da micela zwitteriônica, formada por 61 unidades do surfactante SB3-14 em água ( 23000 moléculas) e obtida por dinâmica molecular foi equilibrada e será a estrutura inicial para a simulação $\mathrm{QM} / \mathrm{MM}$, em que a glicina será descrita pelo método de química quântica (QM) PDDG/PM3 e o restante (micela + águas) por campos de força clássicos (MM). A partir do perfil da 
reação fornecido pelo potencial de força média obtida por amostragem umbrella, em que as janelas são combinadas usando o método de análise ponderada de histogramas, a energia de Gibbs de ativação será determinada.

\section{RESULTADOS E DISCUSSÃO}

Estudamos dois mecanismos possíveis para a reação de descarboxilação da glicina. 0 primeiro consiste na glicina sem a presença da água e nele ocorre a separação da ligação C-C seguida da transferência do próton do grupo $-\mathrm{COOH}$, com a formação do zwitterion e, finalmente, a descarboxilação. No segundo mecanismo temos a reação assistida por água, em que ocorre também a separação da ligação $C$ - C, seguida da transferência do próton, mas este, por sua vez, vai primeiro para a molécula de á gua que então transfere um próton para o grupo $\mathrm{NH}_{2}$. Após isso, ocorre a transferência do próton para o $\mathrm{CH}_{2}$. Esse resultado indica que as águas de hidratação podem desempenhar um papel importante na assistência e catálise da descarboxilação.

Tabela 1. Energia livre da reação $(\mathrm{kcal} / \mathrm{mol})$ de descarboxilação da glicina obtida com o MP2/6$311++\mathrm{G}(2 \mathrm{df}, \mathrm{p})$

\begin{tabular}{ccc}
\hline Glicina neutra & Glicina zwitteriônica & Experimental \\
\hline 48,4 & 50,2 & 46,0
\end{tabular}

A glicina na forma neutra possui barreira de ativação menor do que a da glicina na forma zwitteriônica (Tabela 1), indicando que o mecanismo da saída do próton juntamente com a saída do $\mathrm{CO}_{2}$ deve ser o mecanismo mais provável em água. Esse resultado corrobora a importância das águas de hidratação na assistência e catálise da descarboxilação conforme o perfil de reaç ão para a glicina neutra apresentado na Figura 2 .
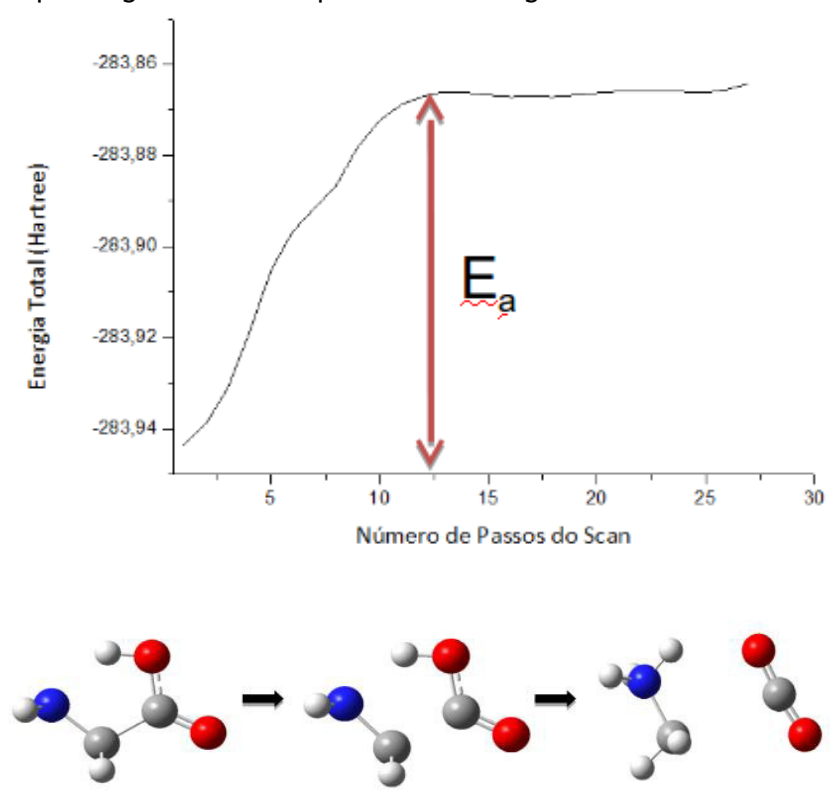

Figura 2. Perfil de reação da glicina neutra (painel superior) calculado com o método MP2/6-311++G(2df,p) e mecanismo da reação de descarboxilação.

Realizamos cálculos de energia para obter os valores correspondentes à energia de ativação da glicina com solvente implícito e com uma molécula de água. Do ponto de vista metodoló gico, o método MP2 $(56,8 \mathrm{kcal} / \mathrm{mol})$ forneceu valores um pouco mais altos que aqueles fornecidos pelo B3LYP $(54,3 \mathrm{kcal} / \mathrm{mol})$.

$\mathrm{Na}$ etapa de dinâmica molecular foi realizada a parametrização da glicina na forma zwitteriônica com cargas RESP obtidas de cálculos "HF/6-31G*//HF/6-31G* - Connolly surface algorithm used in MEP computation - 2 stage RESP fit qwt $=0.0005 / 0.001$ Used in the Cornell et al., Kollman et al., Cheatham et al., Wang et al. \& Hornak et al. AMBER force fields" utilizando o programa RED IV [1]. A caixa de simulação foi construí da com moléculas de água em uma caixa de aproximadamente 50x $50 \times 50 \AA$ Å com a molécula de glicina no centro. A metodologia utilizada foi a de não aquecimento, em que o sistema foi minimizado em 10000 passos e, posteriormente, equilibrado no ensemble NPT a fim de obter a densidade correta para, em seguida, na etapa de produção, calcular as médias das propriedades num ensemble NVT. Utilizamos o barostato e termostato de Berendsen e um tempo total de simulação de $10 \mathrm{~ns}$.

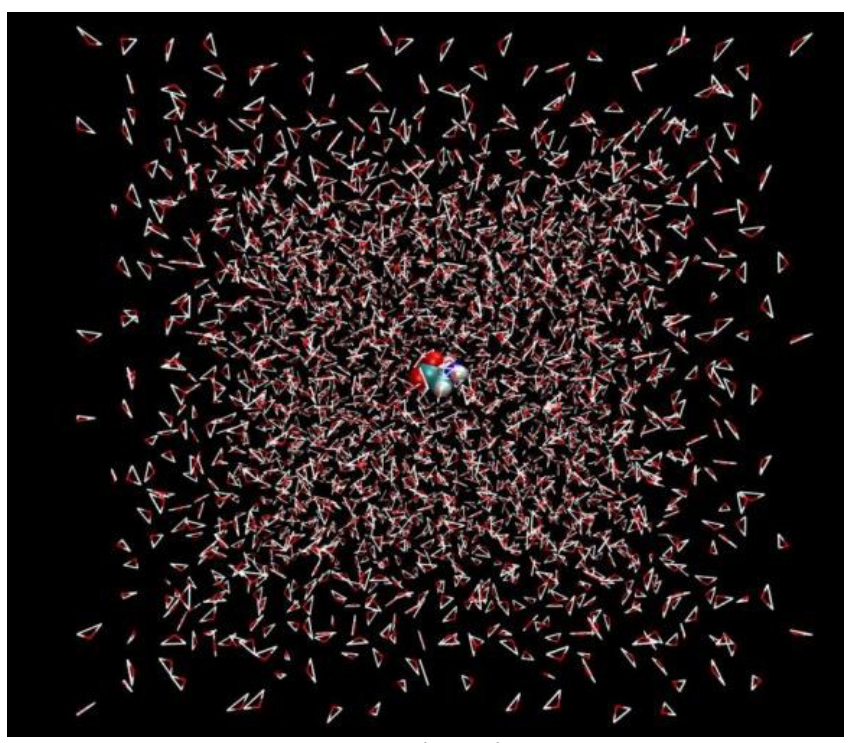

Figura 3. Caixa de simulação da glicina em água após 10 ns de propagação.

Observou-se que a temperatura, a pressão, a energia e o volume se mantiveram em torno das médias. A etapa de equilibraç ão é necessária para manter constantes os valores das propriedades como densidade e temperatura, por exemplo.

As propriedades estruturais foram analisadas pela função de distribuição radial, $g(r)$, das moléculas de água ao redor da glicina. A integral da função de distribuição radial mostra a quantidade de moléculas de água nas camadas de solvatação definidas pelos máximos no gráfico de $g(r)$. A Figura 4 mostra a funções de distribuição radial das moléculas de água (oxigênio da á gua) em relação ao centro de massa da glicina.

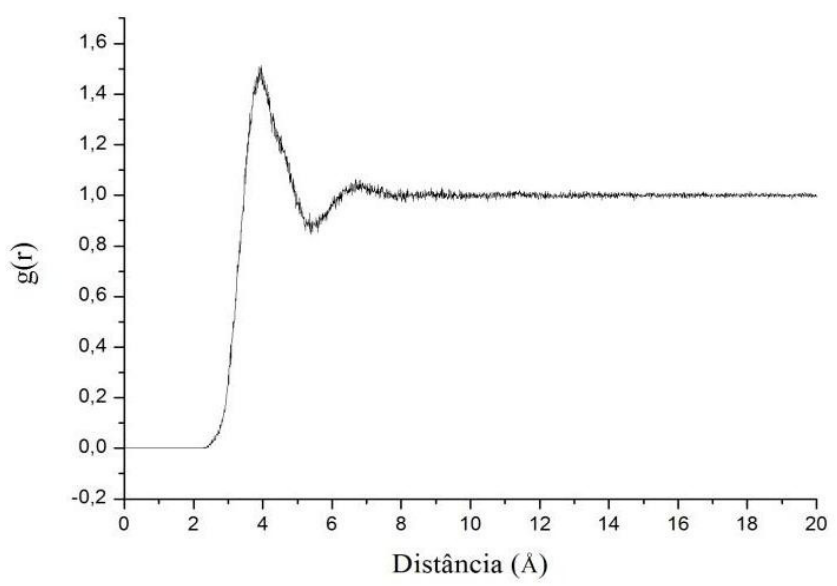




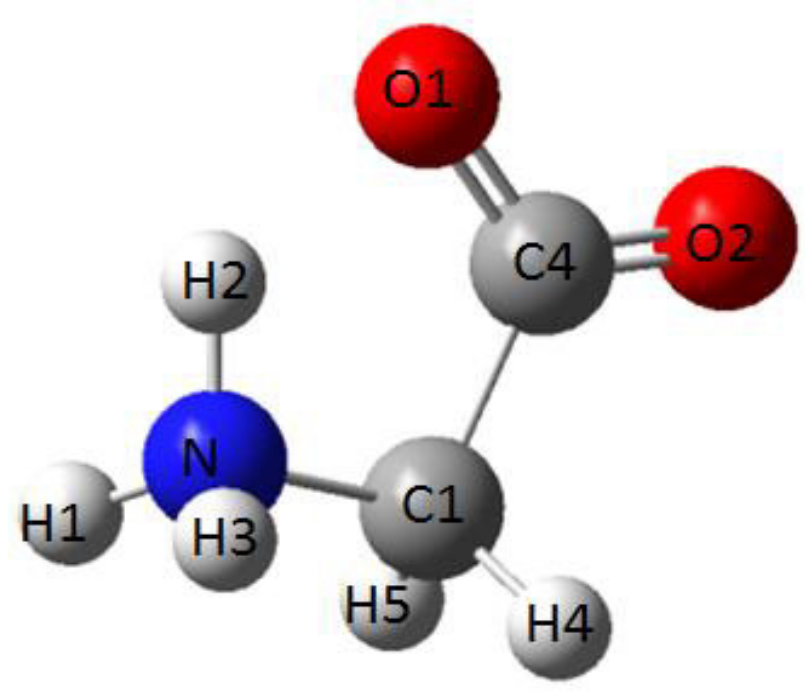

Figura 4. Função de distribuição radial da água e centro de massa da glicina (painel superior) e estrutura da glicina indicando seus átomos.

Das funções de distribuição radial está evidente a presença de duas camadas de solvatação bem definidas. Considerando essas duas camadas de hidratação, o número de molé culas de água que estão solvatando a parte polar da glicina é: $\mathrm{N}$ (glicina)-O(água) = 2,77; 01 (glicina)-O(água) = 2,67 e 02(glicina)O(água) $=2,67$. Ou seja, são aproximadamente iguais a três. Estes valores estão muito próximos daqueles encontrados na literatura [2].

Quando realizamos a simulação da glicina na forma zwitteriônica na superfície da micela SB3-14, observou-se que não ocorre a intrusão da glicina no seu interior (Figura 5). Entretanto, observamos que ocorrem interações com a superfície da micela. Utilizando as configurações geradas na simulação, calculamos o tempo médio de residência da glicina na micela, que foi igual a 0,8 ns. Esse tempo é muito curto e indica que, provavelmente, a micela não deve influenciar significativamente a descarboxilação. Portanto, com base nesses resultados, iniciamos estudos do comportamento da glicina neutra em água e na superfície da micela para verificar o efeito da forma do aminoácido (neutra vs. Zwitteriônica) no tempo de residência.

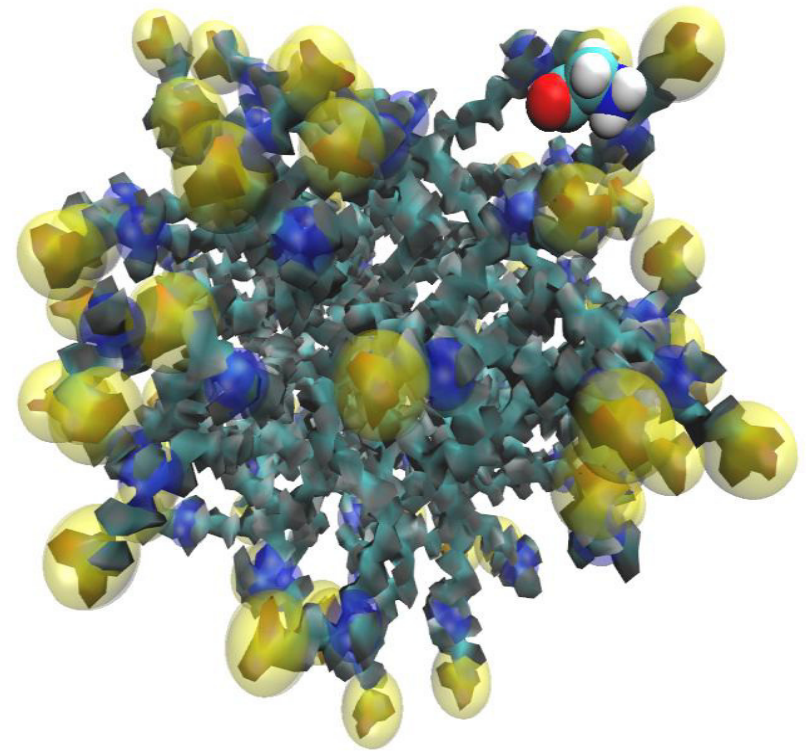

Figura 5. Representação da glicina (zwitteriônica) interagindo com a superfície da micela SB3-14.
Para realizar a dinâmica da glicina neutra, fizemos a parametrização da glicina com cargas RESP obtidas de cálculos $\mathrm{HF} / 6-31 \mathrm{G}^{*} / / \mathrm{HF} / 6-31 \mathrm{G}^{*}$. A partir disso, foi feita a minimização da estrutura e iniciamos a etapa dos cálculos de dinâmica molecular. Observou-se que a temperatura, a pressão, a energia e o volume se mantiveram em torno das médias. Os estudos acerca da interação da glicina neutra com a superfície da micela e os efeitos de sais, especialmente, o perclorato de sódio $\left(\mathrm{NaClO}_{4}\right)$, cujos íons podem se adsorver na interface micela-solução e assim afetar a adsorção da glicina e sua descarboxilação, estão em andamento.

\section{CONCLUSÕES}

Os cálculos das estruturas dos sistemas glicina- $\mathrm{nH}_{2} \mathrm{O}(\mathrm{n}=$ $0,1,2,4)$, na forma neutra, foram concluídos e a partir desses resultados obtemos os perfis da reação que forneceram as energias livres de ativação. A energia livre da reação de descarboxilação foi menor para a forma neutra da glicina, em melhor concordância com o valor experimental, do que para a forma zwitteriônica, indicando que o mecanismo migração do próton juntamente com a saída do $\mathrm{CO}_{2}$ deve ser o mecanismo mais provável em água. Verificamos também que as águas de hidratação podem desempenhar um papel importante na assistência e catálise da descarboxilação. $\mathrm{Na}$ etapa de dinâmica molecular, a caixa computacional equilibrada da glicina em água foi obtida com sucesso e observamos que as propriedades como o número de água de solvatação, por exemplo, estão em concordância com os valores experimentais [3]. Na simulação da glicina zwitteriônica na superfí cie da micela SB3-14 não ocorreu a intrusão da glicina no seu interior. Entretanto, observamos que ocorrem interações com a superfície. A glicina na forma zwitteriônica, por sua vez, apresenta tempo de residência muito curto na superfície da micela e, portanto, a micela não deve influenciar significativamente a sua descarboxilação (não há catálise).

\section{REFERÊNCIAS}

DUPRADEAU, F. et al., Phys. Chem. Chem. Phys., 2010.

ALEXANDROVA, A. N.; JORGENSEN, W. L., J. Phys. Chem. B, 2011.

KAMEDA, Y.; EBATA, H.; USUKI, T.; UEMURA, O.; MISAWA, M. Bull. Chem. Soc. Jpn., 1994. 